\title{
An Adaptive Overcurrent Protection Scheme for Distribution Networks
}

\author{
F. Coffele, C. Booth and A. Dyśko, Member, IEEE
}

\begin{abstract}
Distribution networks are evolving towards the vision of smart grids, with increasing penetration of Distributed Generation (DG), introduction of Active Network Management (ANM) and potentially islanded modes of operation. These changes affect both fault levels and fault current paths and have been demonstrated to compromise the correct operation of the overcurrent protection system.

This paper presents an adaptive overcurrent protection system which automatically amends the protection settings of all overcurrent relays in response to the impact of DG, ANM and islanding operation. The scheme has been developed using commercially available protection devices, employs IEC61850 based communications and has been demonstrated and tested using a Hardware-In-the-Loop (HIL) laboratory facility.

A systematic comparison of the performance of the proposed adaptive scheme alongside that of a conventional overcurrent scheme is presented. This comparison quantifies the decrease in false operations and the reduction of mean operating time that the adaptive system offers.
\end{abstract}

Index Terms-Adaptive protection, network automation, distributed generation, islanded operation, time overcurrent protection.

\section{INTRODUCTION}

$\mathrm{T}$ HE on-going increase in the penetration of DG and the adoption of ANM solutions in distribution networks throughout the world creates a network protection challenge due to the effects on fault levels and fault current paths.

DG introduces an additional source of fault current, which may increase the total fault level within the network, while possibly altering the magnitude and direction of the fault currents seen by specific protection relays. The contribution of one single generating unit is normally not large, but the aggregate effect of many generating units can have a significant impact on fault currents and affect the operation of the overcurrent protection system [1].

ANM solutions, which have been introduced to manage DG, energy storage, loads, circuit breakers and switches to allow voltage control, power flow management, demand side

This work was supported in part by the UK Research Councils' Energy Programme as part of the Supergen FlexNet Consortium, grant no. EP/E04011X/1.

F. Coffele was with the Department of Electronic and Electrical Engineering and he is now with the Power Networks Demonstration Centre, University of Strathclyde, UK. C. Booth and A. Dyśko are with the Department of Electronic and Electrical Engineering, University of Strathclyde, United Kingdom (email: federico.coffele@strath.ac.uk; campbell.d.booth@strath.ac.uk; a.dysko@strath.ac.uk) management, automatic restoration and minimization of power system losses, also affect the fault levels and the fault current paths [2].

Furthermore, as the penetration of DG increases, the islanded operation of certain sections of the distribution network may become beneficial and increase the reliability of power supply to the customers [3]. However, changing between islanded and grid connected modes of operation creates two scenarios with very different fault levels [4].

The combination of DG, ANM and the potential for islanded operation results in network conditions where fault levels and fault current paths change disturbing the operation of the overcurrent relays (OCRs). The authors of [1, 5-8] showed that DG affects the sensitivity and the operating time of the OCRs while the authors of [9] proved that changes in network topology compromise the correct coordination between OCRs. The impact of islanding was analyzed in [4, 10], where the authors assessed the amount of fault level reduction during islanded operation and proved that it causes slow operating times and possible blinding of OCRs.

Solutions to the impact of DG have been presented in [11] where the authors suggest the adoption of distance protection, in [12-14] where the authors propose the use of fault current limiters (FCLs) and in [14-16] where the authors suggest to use adaptive protection. The authors of $[14,15]$ have proposed to use to sets of protection settings, one for DG connected and one for DG not connected to the network, while the authors of [16] have proposed a scheme where the settings of overcurrent protection relays are amended in real time based on the fault level and the DG connection status. A solution that caters for islanded operation has been proposed in [10, 17], where the authors demonstrate how a simple adaptive overcurrent protection scheme with two setting groups, one for grid connected and one for islanded mode of operation may solve the problem. It appears that, as yet, no solution has been proposed to address the impact of ANM systems on network protection.

All of the proposed adaptive overcurrent protection systems in the literature seem concentrate on the solution to a specific protection performance problem and disregard other aspects of future networks that may impact on performance. Therefore, these schemes are somewhat limited, as in future it is likely that DG, ANM and islanded operation will all be factors that will impact on protection.

Accordingly, this paper presents an adaptive overcurrent protection scheme that addresses all of these issues 
simultaneously. The main difficulty in developing a solution to the aggregated problems caused by DG, ANM and islanding is that the number of possible network conditions is very large and it becomes unfeasible to pre-calculate protection settings and establish a manageable number of setting groups which would cover all potential situations. Therefore, the solution proposed in this paper does not use pre-calculated setting groups but rather establishes the optimum protection settings and applies them to the relays directly whenever there is a significant change in the network, either in terms of DG connectivity, grid connected/islanded status or changes implemented by an ANM system.

The paper is organized as follows. Section II describes the proposed adaptive overcurrent protection system, its architecture and its algorithm, section III presents the test case distribution network, and section IV illustrates the hardwarein-the-loop (HIL) simulation environment used to test the proposed solution. Finally, section V presents the simulation results and, through comparison, quantifies the improvements, in terms of dependability, security and mean operation time, offered by the adaptive system over a traditional system.

\section{ADAPTIVE OVERCURRENT PROTECTION SYSTEM}

The adaptive overcurrent protection system has been developed using a three layer architecture illustrated in Fig. 1. The separation of functional layers has been established according to the type of data used and the required response time for each functional group [18].

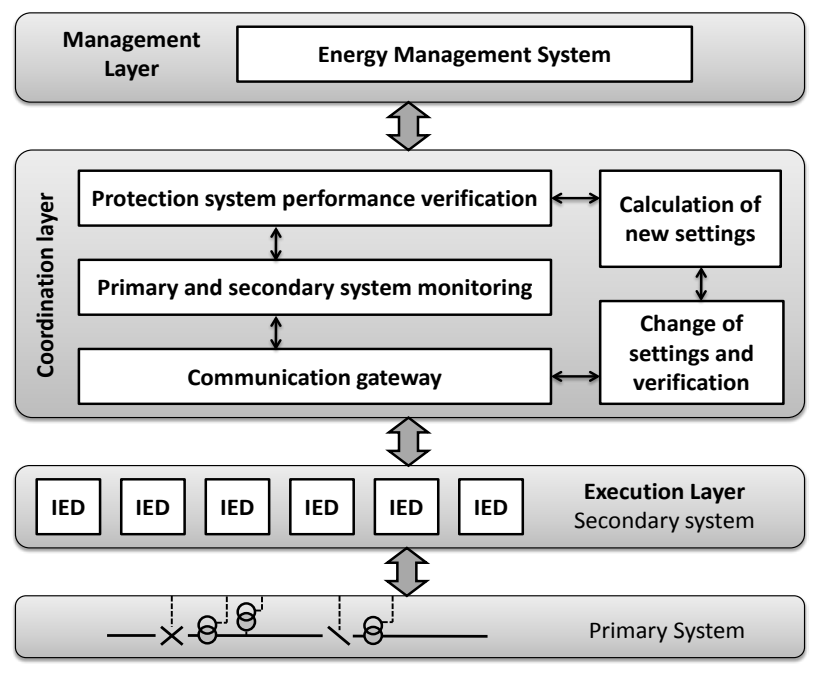

Fig. 1. Adaptive overcurrent protection system architecture

The primary system is at the foot of the diagram, and includes lines, transformers, DG, Circuit Breakers (CBs), Circuit Switches (CSs), Current Transformers (CTs), Voltage Transformers (VTs), etc. Directly above this is the execution layer, which includes the IEDs installed in the network (e.g. OCRs). The interfaces between the first two layers consist of hardwired links for the provision of measurement data and tripping commands or IEC 61850 process bus communication.

The execution layer is connected to the coordination layer, which is responsible for monitoring and coordinating the IEDs. Finally, at the top there is an energy management layer, which is responsible for managing the overall network and communicates to the coordination layer to achieve coordination between adaptive overcurrent protection and ANM. The interface between execution, coordination and management layers are based on communication protocols such as: DNP3, Modbus, IEC60870-5-103 and IEC61850.

The execution layer is composed of OCRs, receiving measurement data from CTs (and in some cases VTs) and tripping $\mathrm{CB}(\mathrm{s})$ when faults are detected that should be cleared by the specific OCR(s).

The execution layer is an autonomous layer, i.e. the tripping decisions are taken locally using local data without any communication with other layers. This means that in case of communication failure between the execution layer and the coordination layer, the overcurrent protection is not affected. If its settings were to be changed remotely, this would not be possible upon failure of the coordination layer or failure of the communication link between these layers, however this would not compromise the overcurrent protection system but would mean that the protection settings are not optimized until the communication is restored.

The implementation of the adaptive protection system is facilitated by the introduction of enhanced functionality to the coordination layer, which includes additional functions that are not present in a traditional protection system. Fig. 2 presents the algorithm of the developed adaptive overcurrent protection system.

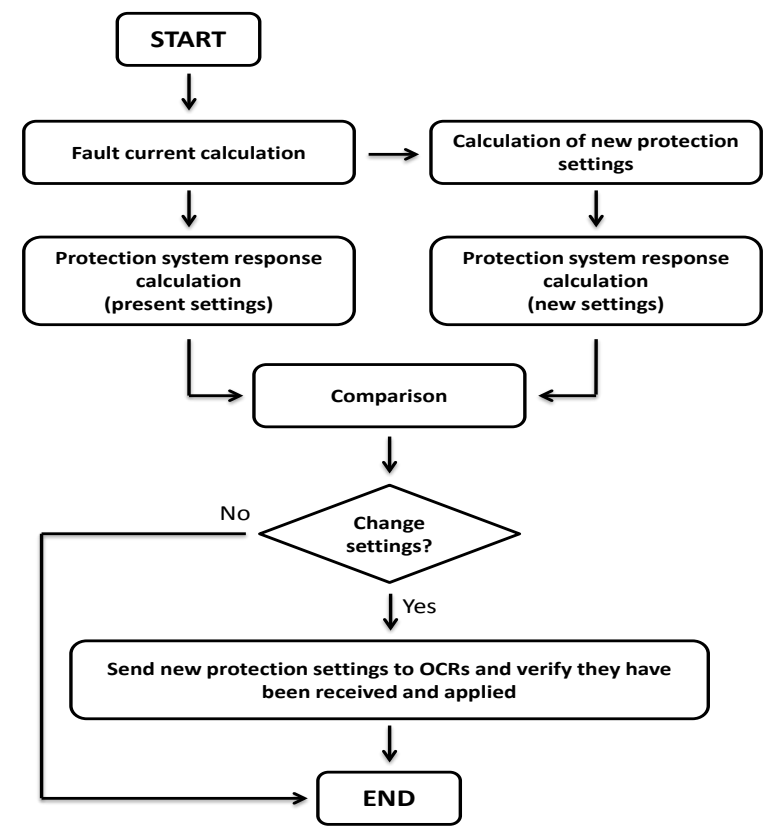

Fig. 2. Adaptive overcurrent protection algorithm

The algorithm is initiated either by the monitoring block in the coordination layer which reacts to changes in the network, or by the energy management system which communicates reconfiguration of the network topology, connectiondisconnection of DG and islanded/grid connected changes.

The adaptive protection system has been implemented using a centralized approach, where setting calculations and modification commands are performed by one processing unit, 
rather than the agent based approach, which typically involves distribution of both processing burden and decision making. The reasons why the centralized approach has been adopted are: simpler implementation in real distribution networks where the present SCADA is centralized, easier commissioning and validation of the centralized solution because compare to the agent based solution.

The following sections explain the individual components of the adaptive overcurrent protection algorithm.

\section{A. Fault current calculation}

Considering the actual network configuration and the status of the DG connection, a series of faults are simulated (usually at the source and remote end of each network section) to calculate the fault current measured by the OCRs for each fault scenario. A program, written in Python 2.7 [19], accesses the IPSA Power [20] fault calculation tool through its application program interface (API), simulates the faults (through instructing IPSA to execute the appropriate simulations) and saves the fault currents that would be measured by each protection device for every simulated fault. These are saved to a fault current matrix F.

$\mathbf{F}=\left[\begin{array}{ccc}I_{f 11} & \cdots & I_{f 1 m} \\ \vdots & \ddots & \vdots \\ I_{f n 1} & \cdots & I_{f n m}\end{array}\right]$

Where $n$ is the number of protection devices and $m$ is the number of simulated faults.

\section{B. Calculation of new protection settings}

New protection settings are calculated considering the present configuration of the network. All OCRs' settings are calculated in a "downstream to upstream" fashion, i.e. starting from the HV/LV transformers' fixed fuse current/time characteristics.

This approach is different from the common approach used by distribution network operators (DNOs) to calculate the protection settings because DNOs normally calculate the protection settings starting from upstream, i.e. grading from the protections at higher voltages and moving downstream; and favors one set of protection settings which would be applicable to all different network configurations. The reason why downstream to upstream calculation method has been adopted instead of the common DNO approach is that it minimizes the protection operating time of the OC protection system for each specific network condition or configuration.

\section{Protection system response calculation}

The protection system response to fault current matrix $\mathrm{F}$ is calculated using both the prevailing protection settings and the new proposed protection settings as calculated in section II.B. The results are saved in the operating time matrices $\mathbf{T}^{0}$ and $\mathbf{T}^{1}$ for the present settings and the new settings respectively.

$\mathbf{T}^{0}=\left[\begin{array}{ccc}t_{11}^{0} & \cdots & t_{1 m}^{0} \\ \vdots & \ddots & \vdots \\ t_{n 1}^{0} & \cdots & t_{n m}^{0}\end{array}\right] ; \quad \mathbf{T}^{1}=\left[\begin{array}{ccc}t_{11}^{1} & \cdots & t_{1 m}^{1} \\ \vdots & \ddots & \vdots \\ t_{n 1}^{1} & \cdots & t_{n m}^{1}\end{array}\right]$

Where $n$ is the number of protection devices being considered; and $m$ is the number of simulated faults.

\section{Comparison of relative performance and setting application decision}

The protection system responses (both with the prevailing settings and the new settings) are analyzed to establish if improvement can be achieved through setting modification. If the new protection settings improve the performance, the decision is made to apply the new settings, otherwise no further action is taken.

To compare the protection system responses $\mathrm{T}^{0}$ and $\mathrm{T}^{1}$, a dedicated algorithm has been designed (implemented in Python 2.7) which analyses both matrices in order to:

1. Verify that the operation time of each OCR is within the limits specified in the protection policy;

2. Verify the grading margin between protection devices;

3. Calculate the mean operation time.

For example, considering a simple circuit in Fig. 3, step 1 verifies that for faults 4 and 5 the operation time of OCR C is shorter than the limits specified in the protection requirements (e.g. an operation time limit of $1 \mathrm{~s}$ is typically used in utility protection policies). Step 2 verifies that the difference of the operation time between OCR C and the back-up protection OCR $\mathrm{B}$ is greater than the minimum grading margin specified in the protection requirements (an example grading margin is $0.3 s$ in a typical utility protection policy).

Steps 1 and 2 are repeated for a series of simulated faults at different locations (the source and remote end of each feeder section) as shown in Fig. 3.

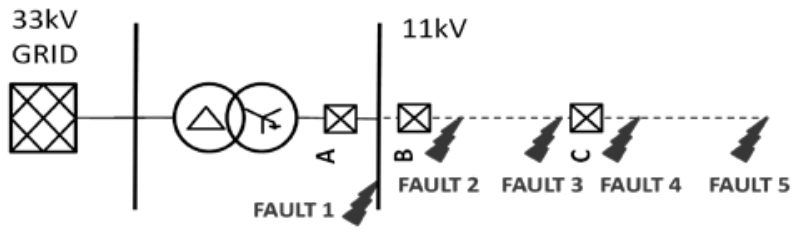

Fig. 3. Fault locations for the protection system response comparison

The first two steps have a higher priority with respect to the third step, therefore if $\mathrm{T}^{1}$ does not pass the two verification steps but $\mathrm{T}^{0}$ does, the proposed new settings are discarded, while if $\mathrm{T}^{0}$ does not pass the two verification steps and $\mathrm{T}^{1}$ does, the proposed new settings are applied, without the third verification step.

If both $\mathbf{T}^{0}$ and $\mathbf{T}^{1}$ pass the first and the second verification, the third step is the comparison of the mean operation times obtained from $\mathbf{T}^{0}$ and $\mathbf{T}^{1}$ according to equations (3) and (4).

$\mathrm{t}_{\text {mean }}^{0}=\frac{1}{n m} \sum_{i=1}^{n}\left(\sum_{j=1}^{m} t_{i j}^{0}\right)$
$\mathrm{t}_{\text {mean }}^{1}=\frac{1}{n m} \sum_{i=1}^{n}\left(\sum_{j=1}^{m} t_{i j}^{1}\right)$

Finally, the two mean operation times are compared using equation (5), and if the condition is satisfied, the new protection settings are applied. 
$\mathrm{t}_{\text {mean }}^{0}-\mathrm{t}_{\text {mean }}^{1}>\Delta t_{m}$

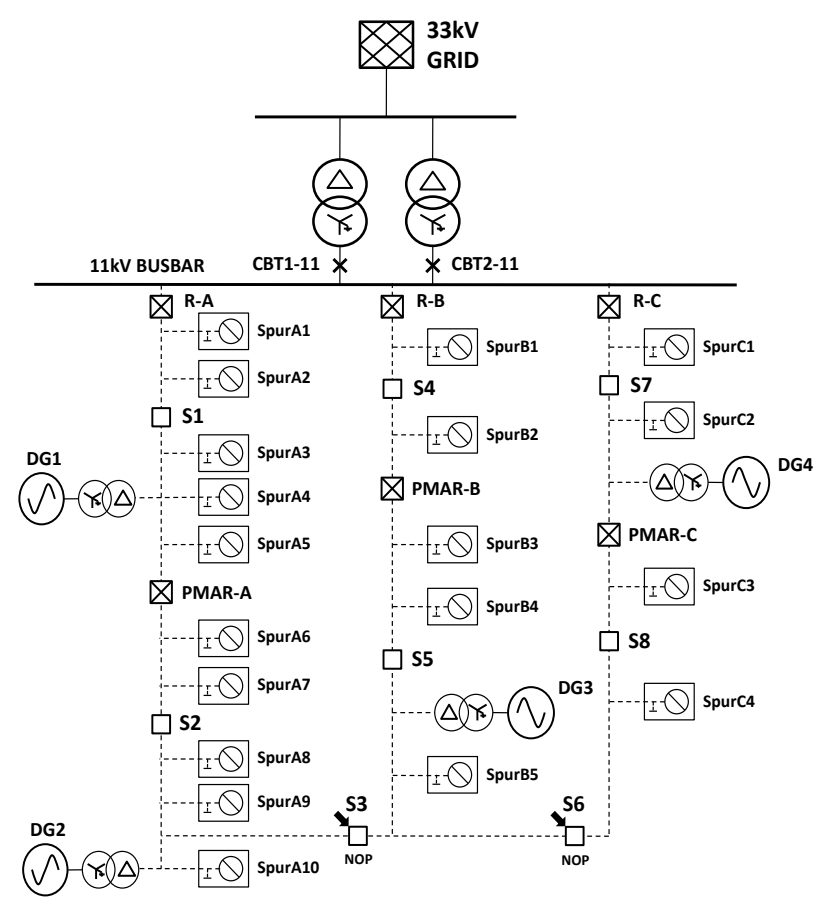

Fig. 4. Distribution network test case diagram

where $\Delta t_{m}$ the minimum difference of the mean times below which the new settings are not applied because the benefit in changing the protection settings would be negligible compared to the risk of fault occurring during the change process.

\section{E. Applying new protection settings and verification}

The final step of the algorithm is to send the new protection settings to the OCRs. This is achieved using IEC61850 communication in two stages. The first stage involves sending the settings, while the second phase involves reading the settings in order to verify that they have been correctly applied.

An IEC61850 compliant protection relay can facilitate two approaches that will enable the application of variable protection settings:

The first approach is based on the employment of protection setting groups. Typically, four or more protection setting groups can be defined and the adaptive protection system can select the group that represents the closest match to the specific calculated protection settings.

The second approach does not employ predefined protection setting groups. Each specific protection setting (e.g. pick up current, time multiplier, etc.) is accessible for modification and the adaptive protection system can write the calculated protection settings on an individual basis.

The first approach has the advantage to avoid the risk of applying wrong protection settings which might cause false tripping or no operation of the protection system during faults while the advantage of the second approach is that it allows more flexibility.

\section{DISTRIBUTION TEST CASE NETWORK MODEL}

The test case network used in this paper is an $11 \mathrm{kV}$ overhead rural distribution network, the "OHA Network", as specified in the United Kingdom Generic Distribution Network (UKGDS)[21]. Fig. 4 depicts the topology of the network which consists of three main feeders and several relatively long spurs.

Both 33/11 kV transformers are rated at 12MVA, with 8.5\% per-unit reactance, delta-star winding configuration and solid earth connections on the $11 \mathrm{kV}$ side. The lengths of feeders A, $\mathrm{B}$ and $\mathrm{C}$ are $8.5 \mathrm{~km}, 3.5 \mathrm{~km}$ and $2.2 \mathrm{~km}$ respectively. Feeder $\mathrm{A}$ is rated at 400A (7.62MVA), while feeders $\mathrm{B}$ and $\mathrm{C}$ both have a rating of $250 \mathrm{~A}$ (4.76MVA).

The protection system has been designed to accurately represent present-day networks and adheres to a protection policy that has been supplied by a UK distribution network operator (DNO). As shown in Fig. 4, each feeder is protected by a multi-shot circuit breaker/recloser at the source end, and by a Pole Mounted Auto-Recloser (PMAR) situated at approximately $50 \%$ along the feeder. Spurs are connected to the main feeder through spur sectionalizers rather than via fuses, due to the prevailing trend within DNOs to substitute fuses with spur sectionalizers in modern and future distribution networks.

\section{LABORATORY IMPLEMENTATION AND SIMULATION}

The developed adaptive protection solution has been implemented and demonstrated in a HIL laboratory environment, shown in Fig. 5, in order to verify its effectiveness and compare its performance with a traditional overcurrent protection system.

The real time digital simulator (RTDS) is used to simulate the primary system behavior in real time during normal and faulty conditions. The output currents of the simulated CTs are amplified using slave amplifiers to inject the OCRs, which operate as if they were connected to a real distribution network and, in the presence of faults, send tripping signals using IEC 61850 GOOSE messaging. The tripping signals are received by the RTDS as an input to the simulation, closing the simulation loop. 


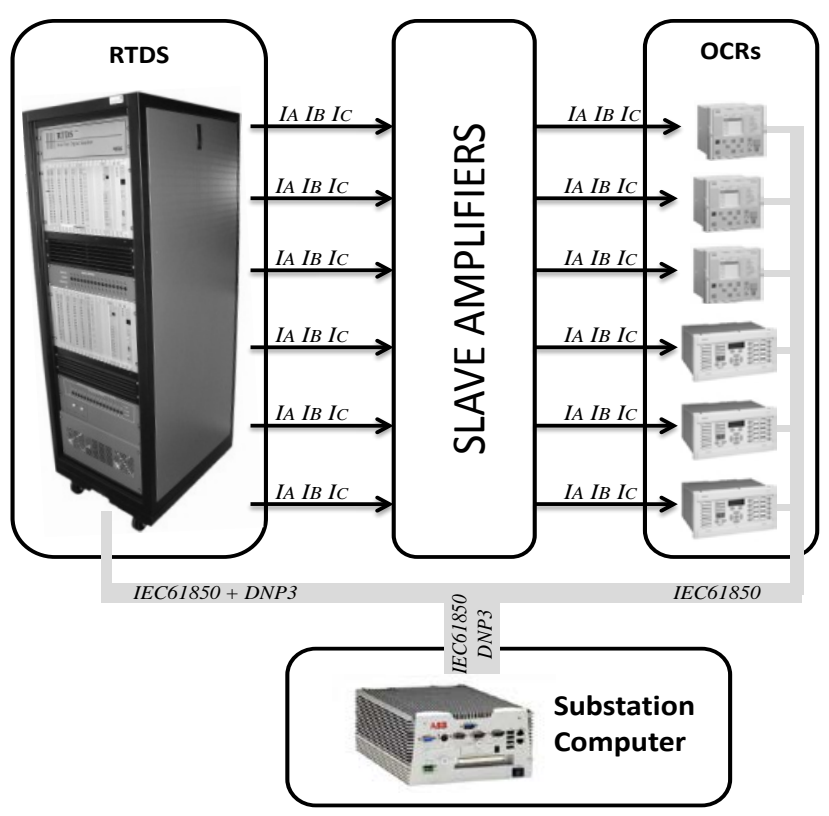

TABLE I

NETWORK SCENARIOS

\begin{tabular}{ccccc}
\hline \hline N. & $\begin{array}{c}\text { 33kV fault } \\
\text { level } \\
\text { (MVA) }\end{array}$ & $\begin{array}{c}\text { Substation } \\
\text { transformers } \\
\text { in service }\end{array}$ & $\begin{array}{c}\text { Normally } \\
\text { Open } \\
\text { Points }\end{array}$ & $\begin{array}{c}\text { DG } \\
\text { units } \\
\text { in service }\end{array}$ \\
\hline 1 & 300 & 2 & S3, S6 & No \\
2 & 300 & 2 & S1, S6 & No \\
3 & 300 & 2 & S4, S6 & No \\
4 & 300 & 2 & S5, S7 & No \\
5 & 300 & 2 & S3, S6 & Yes \\
6 & 300 & 2 & S1, S6 & Yes \\
7 & 300 & 2 & S4, S6 & Yes \\
8 & 300 & 2 & S5, S7 & Yes \\
9 & 100 & 1 & S3, S6 & Yes \\
10 & 100 & 1 & S1, S6 & Yes \\
11 & 100 & 1 & S4, S6 & Yes \\
12 & 100 & 1 & S5, S7 & Yes \\
13 & NC & NC & S3, S6 & Yes \\
14 & NC & NC & S1, S6 & Yes \\
15 & NC & NC & S4, S6 & Yes \\
16 & NC & NC & S5, S7 & Yes \\
\hline \hline
\end{tabular}

Fig. 5. HIL laboratory testing environment

A DNP3 master installed on the substation computer is used to communicate with the RTDS to gather periodically status information of CBs, PMARs, network switches, etc. This data is then used by the adaptive overcurrent protection software installed in the substation computer to monitor the network and detect changes which initiate the adaptive algorithm shown in Fig. 2.

When the adaptive protection software is required to change the protection settings of one or more OCRs, this is achieved using an IEC61850-8 master installed in the substation computer to communicate with the OCRs.

In order to demonstrate the effectiveness of the developed adaptive overcurrent protection system, a number of scenarios have been simulated (refer to Table I).

The adaptability of the protection system is stimulated by applying various changes to the network configuration. After each change a set of pre-defined faults are simulated to verify the protection system performance.

\section{A. Network scenarios}

Scenarios summarized in Table I have been generated to include the following stimuli to the adaptive overcurrent protection system:

- Change of fault level due to changes of fault level at $33 \mathrm{kV}$ and the number of in-service transformers at the $33 / 11 \mathrm{kV}$ distribution substation. Normally both transformers are in operation, but in some cases one may be disconnected.

- Islanded operation of the $11 \mathrm{kV}$ network, which may be permissible if appropriate DG units are in service.

- Change of $11 \mathrm{kV}$ distribution network topology, which can be varied by shifting the normally open points (NOP) as necessary.

- Connection/disconnection of the DG units.

\section{B. Fault simulations}

In order to verify the response of the adaptive overcurrent protection system, a series of pre-defined faults have been simulated for each network scenario, in twelve different fault locations, as shown in Fig. 6.

The faults simulated at each location include:

- Eleven phase to phase faults with a fault resistance between $0 \Omega$ and $10 \Omega(0 \Omega, 1 \Omega, 2 \Omega$, etc.);

- Eleven phase to earth faults with a fault resistance between $0 \Omega$ and $100 \Omega(0 \Omega, 10 \Omega, 20 \Omega$, etc.).

All faults have been simulated twice, to test both the traditional overcurrent protection system as well as the adaptive overcurrent protection system.

\section{Simulation RESUltS}

The protection system response of the developed adaptive scheme and of the conventional overcurrent protection system with fixed protection settings has been recorded for phase to phase and phase to earth faults described in section IV.B.

Fig. 7 shows the measured operating times of the conventional and adaptive overcurrent protection systems for all of the 2112 simulated phase faults. The responses are

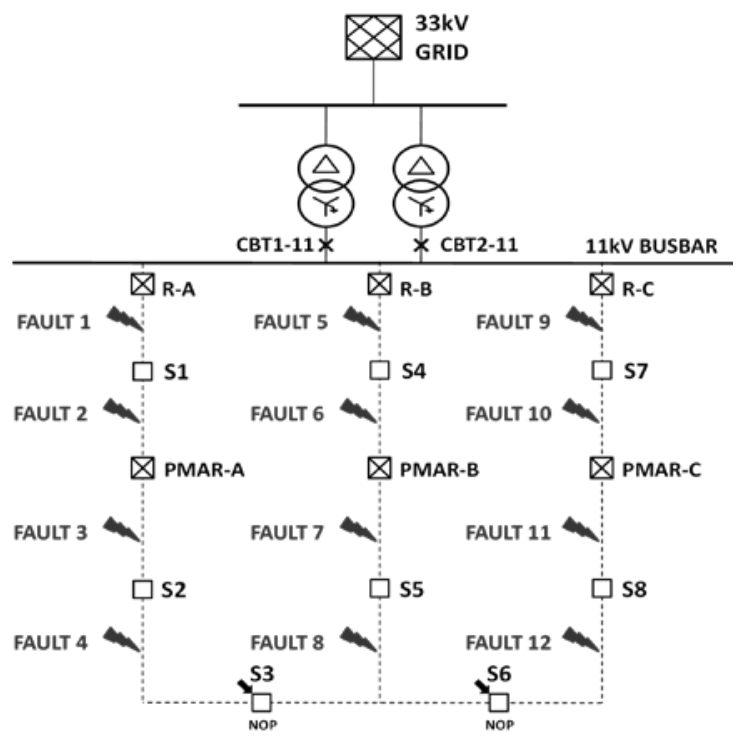

Fig. 6 Fault locations for the HIL simulation 


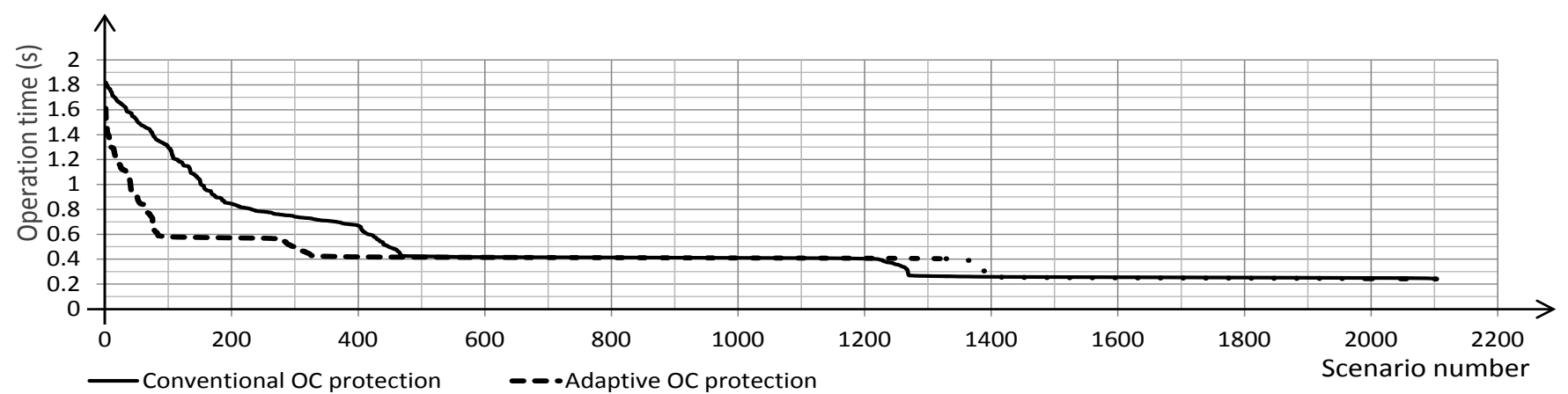

Fig. 7 Measured operating time of conventional and adaptive protection during phase to phase faults

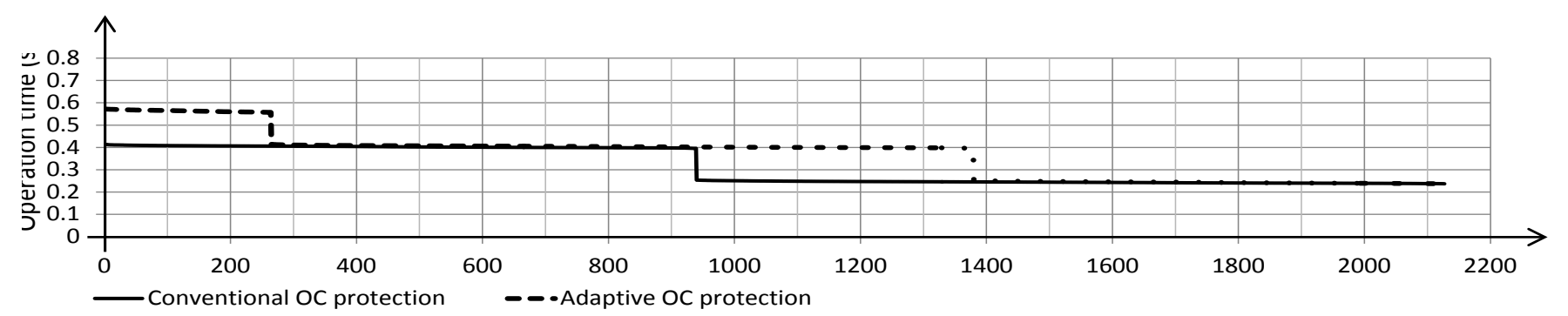

Fig. 8 Measured operating time of conventional and adaptive protection during phase to earth faults

ordered according to the tripping time starting from the longest. For the first 456 longest faults, the operation of the adaptive overcurrent protection is faster than the conventional IDMT overcurrent protection system, reducing the number of operating times longer than $1 \mathrm{~s}$ from $7.15 \%$ to $1.81 \%$ of the total number of simulated faults.

For all of the other faults, there is no appreciable difference in tripping time, because the delay times of the DTL characteristics are identical. The only exception is for the fault scenarios between 1214 and 1388, where the adaptive overcurrent protection system has a slower tripping time. This is due to the correction of the DTL overcurrent protection settings to guarantee correct coordination between the OCRs when the network topology changes.

Fig. 8 shows the operating time of conventional and adaptive overcurrent protection system measured for the 2112 simulated earth faults also ordered according to the tripping time and starting from the longest response. Note that, as with the phase fault results, the operation of the adaptive protection system is slower for some faults, which is necessary to ensure correct coordination between OCRs as the network topology changes. The increase in operating time is not significant and it is not considered to be a problem since the maximum operating time is of $0.572 \mathrm{~s}$. From the results, it is clear that the adaptive protection can be marginally slower in some cases for both phase and earth faults. Nevertheless, overall improvement in performance is achieved as coordination problems are avoided by the modification of settings and increased time response to some of the simulated faults.

The analysis of the results revealed that the adaptive overcurrent protection system has improved selectivity and sensitivity with respect to conventional overcurrent protection, for example, when the network topology is changed. It also provides improved coordination with the DG interface protection compared to conventional overcurrent protection. Selected cases are presented below to demonstrate some of these advantages.

TABLE II

PROTECTION SETTINGS NETWORK SCENARIO 1

\begin{tabular}{cccccccc}
\hline \hline & \multicolumn{3}{c}{ IDMT Phase OC } & \multicolumn{2}{c}{ DTL Phase OC } & \multicolumn{2}{c}{ Earth DTL OC } \\
& CH & Iset & TMS & Iset & DTL & Iset & DTL \\
\hline AR-A & SI & 400 & 0.16 & 1000 & 0.32 & 30 & 0.32 \\
PMAR-A & SI & 250 & 0.1 & 625 & 0.16 & 30 & 0.16 \\
AR-B & SI & 350 & 0.2 & 875 & 0.32 & 30 & 0.32 \\
PMAR-B & SI & 220 & 0.1 & 550 & 0.16 & 30 & 0.16 \\
AR-C & SI & 300 & 0.28 & 750 & 0.32 & 30 & 0.32 \\
PMAR-C & SI & 180 & 0.1 & 450 & 0.16 & 30 & 0.16 \\
\hline \hline
\end{tabular}

TABLE III

PROTECTION SETTINGS NETWORK SCENARIO 3

\begin{tabular}{cccccccc}
\hline \hline & \multicolumn{3}{c}{ IDMT Phase OC } & \multicolumn{2}{c}{ DTL Phase OC } & \multicolumn{2}{c}{ Earth DTL OC } \\
& CH & Iset & TMS & Iset & DTL & Iset & DTL \\
\hline AR-A & SI & 400 & $\mathbf{0 . 1 7}$ & 1000 & $\mathbf{0 . 4 8}$ & 30 & $\mathbf{0 . 4 8}$ \\
PMAR-A & SI & 250 & $\mathbf{0 . 1 2}$ & 625 & $\mathbf{0 . 3 2}$ & 30 & $\mathbf{0 . 3 2}$ \\
AR-B & SI & $\mathbf{2 5 0}$ & $\mathbf{0 . 1}$ & $\mathbf{6 2 5}$ & $\mathbf{0 . 1 6}$ & 30 & $\mathbf{0 . 1 6}$ \\
PMAR-B & SI & $\mathbf{1 6 0}$ & 0.1 & $\mathbf{4 0 0}$ & 0.16 & 30 & 0.16 \\
AR-C & SI & 300 & 0.28 & 750 & 0.32 & 30 & 0.32 \\
PMAR-C & SI & 180 & 0.1 & 450 & 0.16 & 30 & 0.16 \\
\hline \hline
\end{tabular}

TABLE IV

PROTECTION SETTINGS NETWORK SCENARIO 13

\begin{tabular}{cccccccc}
\hline \hline & \multicolumn{3}{c}{ IDMT Phase OC } & \multicolumn{2}{c}{ DTL Phase OC } & \multicolumn{2}{c}{ Earth DTL OC } \\
& CH & Iset & TMS & Iset & DTL & Iset & DTL \\
\hline AR-A & SI & $\mathbf{2 4 0}$ & $\mathbf{0 . 1 2}$ & $\mathbf{6 0 0}$ & 0.32 & 30 & 0.32 \\
PMAR-A & SI & $\mathbf{1 5 0}$ & 0.1 & $\mathbf{3 7 5}$ & 0.16 & 30 & 0.16 \\
AR-B & SI & $\mathbf{2 1 0}$ & $\mathbf{0 . 1 8}$ & $\mathbf{5 2 5}$ & 0.32 & 30 & 0.32 \\
PMAR-B & SI & $\mathbf{1 3 0}$ & 0.1 & $\mathbf{3 2 5}$ & 0.16 & 30 & 0.16 \\
AR-C & SI & $\mathbf{1 8 0}$ & $\mathbf{0 . 1 9}$ & $\mathbf{4 5 0}$ & 0.32 & 30 & 0.32 \\
PMAR-C & SI & $\mathbf{1 0 0}$ & 0.1 & $\mathbf{2 5 0}$ & 0.16 & 30 & 0.16 \\
\hline \hline
\end{tabular}




\section{A. Impact of network automation}

Considering scenarios 1 and 3 in Table I, when the network switches from one scenario to another, the adaptive overcurrent protection system calculates the protection settings for the new scenario and applies them to the OCRs. Table II and Table III report the automatically calculated protection settings for the two scenarios.

The difference between scenario 1 and scenario 3 is the change of network configuration, i.e. the fact that the NOP is shifted from S3 to S4. The change of network topology affects both fault current magnitude and path in case of faults in feeder $\mathrm{A}$ and $\mathrm{B}$. The new protection settings are therefore different for OCRs AR-A, PMAR-A, AR-B and PMAR-B as can be observed by comparing Tables II and III. The numbers in bold indicate the modified protection settings.

Without the developed adaptive protection system, i.e. using fixed protection settings, the operation speed and correct selectivity of the overcurrent protection system are affected. For example, a $0 \Omega$ phase to phase fault between PMAR-B and S4 (fault 6 in Fig. 6) causes the operation of both PMAR-A and PMAR-B, as shown in Fig. 9, which causes the unnecessary disconnection of all loads connected between PMAR-A and PMAR-B. With the adaptive system, the problem of miss-coordination between PMAR-A and PMAR$\mathrm{B}$ is solved in this particular example, as shown in Fig. 10.

When considering the complete population of simulated faults, it is clear that the instances of false tripping have been reduced from $4.72 \%$ to $1.61 \%$ (improved security).

\section{B. Impact of DG}

Consider scenario 5 in Table I, DG1, DG2, DG3 and DG4 are connected to the network. The presence of DG increases the fault level, changes the magnitudes and paths of fault currents and therefore may cause false tripping and affect the coordination between OCRs.

An example of false tripping is when, in scenario 5 , there is a fault on feeder B (fault 4) and the AR-A trips simultaneously with AR-B. This is due to the fault current contribution of DG1 and DG2 to fault 5 and this situation is typical when DNOs adopt DTL instead of IDMT overcurrent protection. To overcome this issue, the protection settings calculated by the overcurrent protection software, and presented in Tables II, III and IV, are based on IDMT overcurrent protection plus DTL overcurrent protection for relatively higher fault currents. The DTL pickup current for the protection on each feeder is higher than the total fault current contributions from DGs on the protected feeders to faults located in adjacent feeders.

Another example of false tripping is due to incorrect overload tripping of OCRs. This happens with traditional overcurrent protection when the network topology is changed and DG creates a load flow that is higher than the tripping current of an OCR. For example, if the network switches to scenario 8, all DG units are connected to feeder 1 and with fixed protection settings may cause false tripping of PMAR-A. While by automatically adapting the protection settings (within the thermal limits of the network), i.e. increasing the pickup current of PMAR-A to be higher than sum of the maximum generation from DG2, DG3 and DG4, the problem of false tripping may be overcome.

\section{Impact of islanded operation}

Considering the case when the network changes from grid connected to islanded operation, the fault level changes significantly. For example, from scenario 1 to 10 in Table I, the fault level at the $11 \mathrm{kV}$ bus bar decreases from $6.8 \mathrm{kA}$ to $1.7 \mathrm{kA}$, affecting both speed and sensitivity of the protection, potentially leading to slow or even non operation during a fault. This may lead to disconnection of the DG supplying the network due to DG interface protection operation.

Dependability of the protection system can be improved in this case by the appropriate adaptation of settings. For example, for a fault downstream of PMAR-B, fault 8, in scenario 10 , the standard overcurrent protection system is too slow as shown in Fig. 11, and therefore the DG interface

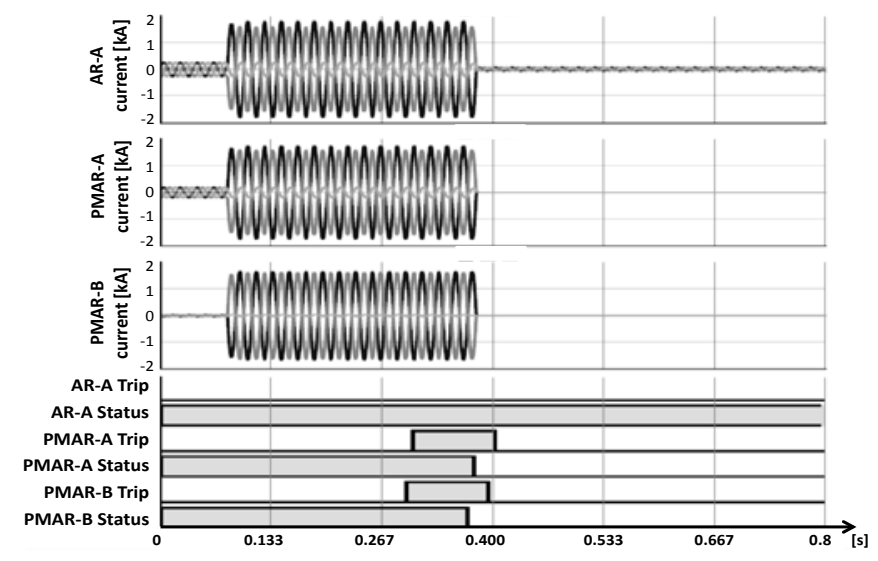

Fig. 9 Fault 6 in scenario 3 without adaptive over-current protection

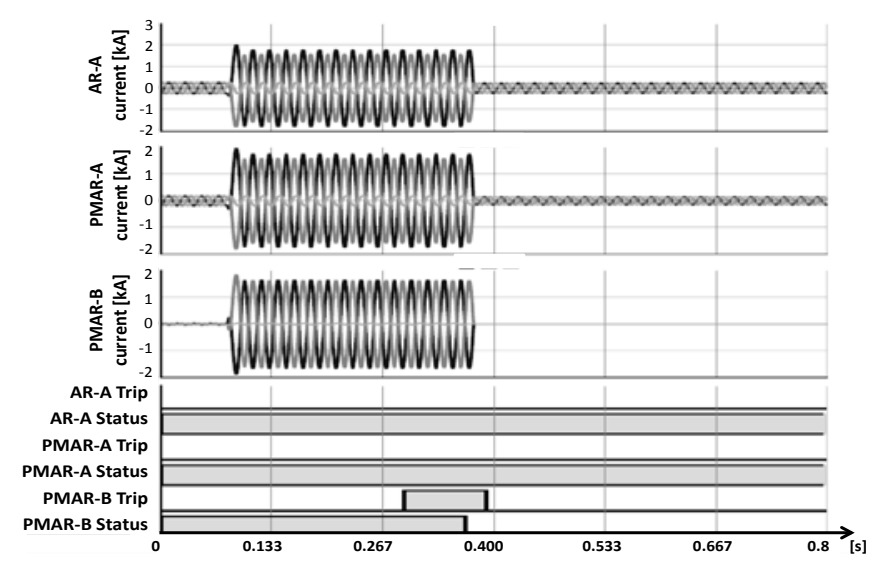

Fig. 10 Fault 6 in scenario 3 with adaptive over-current protection 


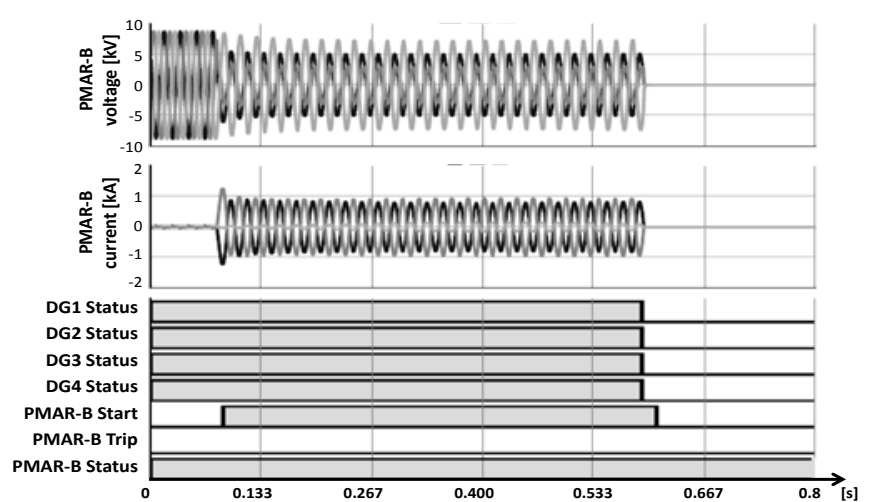

Fig. 11 Fault 8 in scenario 13 without adaptive over-current protection

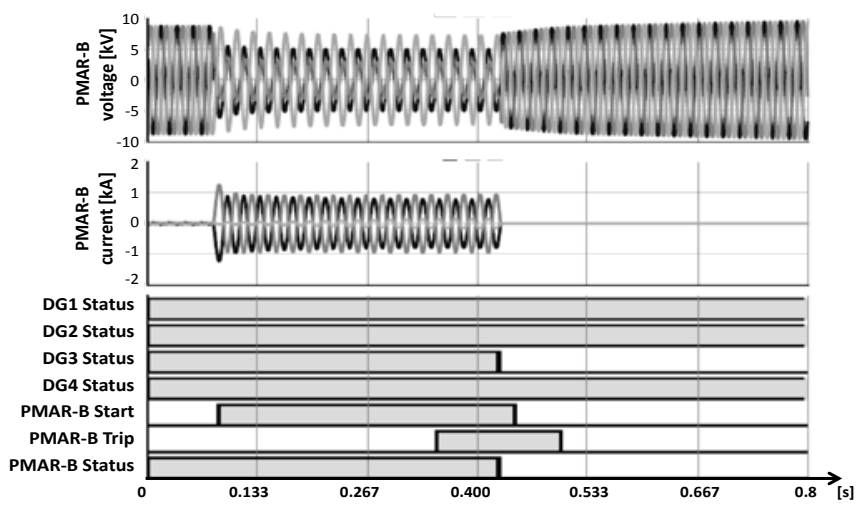

Fig. 12. Fault 8 in scenario 13 with adaptive over-current protection

protection trips before the overcurrent protection system isolates the faulted zone.

In the simulation the interface of the DG has been configured with protection using settings defined in G59/2[22]. Fig. 11 shows that the under voltage protection trips the DG units after $0.5 \mathrm{~s}$.

Simulating the same fault, but with the developed adaptive overcurrent protection system (which changes the protection settings to the values reported in Table IV as soon as the network changes configuration), the overcurrent protection operation is faster as shown in Fig. 11. With the overcurrent protection operating faster, the voltage sags has a shorter duration (as shown in Fig. 12) and DG1, DG2 and DG3 are not disconnected, but continue to supply the loads.

\section{CONCLUSION}

It is clear that the widespread introduction of DG and ANM schemes and the potential for islanded operation of networks in the future will present significant challenges to existing network protection. This paper has illustrated how an adaptive protection scheme can act to address many of these problems and has demonstrated its implementation using a realistic model of an actual distribution network with commercially available hardware and communication schemes.

The novelty of the adaptive overcurrent protection system proposed in this paper is in its algorithm, which differs from other adaptive protection solutions presented in the literature in terms of its possession of higher flexibility and comprehensive coverage of all events that may influence the behavior of the protection system. The algorithm has been explained in detail, focusing on the protection settings calculation technique and the protection system response evaluation.

The proposed adaptive protection solution is more flexible with respect to other solutions presented in the literature, which are largely based on pre-calculated protection settings and settings groups. The limitations of using setting groups with pre-calculated settings is overcome by calculating the optimal protection settings in real time and applying them, after verification of their effectiveness using model-based performance evaluation, to the OCRs when the network status changes.

\section{REFERENCES}

[1] P. P. Barker and R. W. De Mello, "Determining the impact of distributed generation on power systems. I. Radial distribution systems," in IEEE Power Engineering Society Summer Meeting, 2000, pp. 1645-1656.

[2] J. Mutale, "Benefits of Active Management of Distribution Networks with Distributed Generation," in IEEE PES Power Systems Conference and Exposition, 2006, pp. 601-606.

[3] J. C. Gomez and M. M. Morcos, "Distributed generation: Exploitation of islanding operation advantages," presented at the Transmission and Distribution Conference and Exposition: Latin America, 2008.

[4] A. A. Chowdhury, D. O. Koval, and S. M. Islam, "Islanding operation of distributed generators in active distribution networks," presented at the Universities Power Engineering Conference, 2008. UPEC 2008. 43rd International, 2008.

[5] C. Mozina, "Impact of Green Power Distributed Generation," Industry Applications Magazine, IEEE, vol. 16, pp. 55-62, 2010.

[6] K. Kauhaniemi and L. Kumpulainen, "Impact of distributed generation on the protection of distribution networks," in Eighth IEE International Conference on Developments in Power System Protection, 2004, pp. 315-318.

[7] A. Girgis and S. Brahma, "Effect of distributed generation on protective device coordination in distribution system," in Large Engineering Systems Conference on Power Engineering, 2001, pp. 115-119.

[8] K. L. Butler-Purry and M. Marotti, "Impact of Distributed Generators on Protective Devices in Radial Distribution Systems," in Transmission and Distribution Conference and Exhibition, 2005/2006 IEEE PES, 2006, pp. 87-88.

[9] M. S. Sachdev, T. S. Sidhu, and B. K. Talukdar, "Topology detection for adaptive protection of distribution networks," in Energy Management and Power Delivery, 1995. Proceedings of EMPD '95., 1995 International Conference on, 1995, pp. 445-450.

[10] P. Mahat, C. Zhe, B. Bak-Jensen, and C. L. Bak, "A Simple Adaptive Overcurrent Protection of Distribution Systems With Distributed Generation," IEEE Transactions on Smart Grid, vol. 2, pp. 428-437, 2011.

[11] I. Chilvers, N. Jenkins, and P. Crossley, "Distance relaying of $11 \mathrm{kV}$ circuits to increase the installed capacity of distributed generation," IEE Proceedings Gneration, Transmission and Distribution, vol. 152, pp. 40-46, 2005.

[12] G. Tang and M. R. Iravani, "Application of a fault current limiter to minimize distributed generation impact on coordinated relay protection," presented at the Internetional conference on Power System Transients, Motreal, CANADA, 2005.

[13] A. Agheli, H. A. Abyaneh, R. M. Chabanloo, and H. H. Dezaki, "Reducing the impact of DG in distribution networks protection using fault current limiters," in 4th International Power Engineering and Optimization Conference, 2010, pp. 298-303.

[14] W. El-khattam and T. S. Sidhu, "Resolving the impact of distributed renewable generation on directional overcurrent relay coordination: a case study," Renewable Power Generation, IET, vol. 3, pp. 415-425, 2009.

[15] M. Baran and I. El-Markabi, "Adaptive over current protection for distribution feeders with distributed generators," in IEEE PES Power Systems Conference and Exposition, 2004, pp. 715-719. 
[16] N. Schaefer, T. Degner, A. Shustov, T. Keil, and J. Jaeger, "Adaptive protection system for distribution networks with distributed energy resources," presented at the 10th IET International Conference on Developments in Power System Protection (DPSP 2010), 2010.

[17] H. Cheung, A. Hamlyn, Y. Cungang, and R. Cheung, "Network-based Adaptive Protection Strategy for Feeders with Distributed Generations," in IEEE Canada Electrical Power Conference, 2007, pp. 514-519.

[18] I. Abdulhadi, F. Coffele, A. Dysko, C. Booth, and G. Burt, "Adaptive protection architecture for the smart grid," in Innovative Smart Grid Technologies (ISGT Europe), 2011 2nd IEEE PES International Conference and Exhibition on, 2011, pp. 1-8.

[19] Python. (1st October 2012). Python Software 2.7. Available: http://www.python.org

[20] TNEI. (1st October 2012). IPSA Power Software. Available: http://www.ipsa-power.com/software

[21] DTI, Ed., United Kingdom Generic Distribution System (UKGDS). DTI (UK), 2005, p.^pp. Pages.

[22] ENA, ER G59/2 Recommendations For The Connection Of Generating Plant To The Distribution Systems Of Licensed Distribution Network Operators, 2010.

Federico Coffele received the B.Eng and M.Eng.from the University of Padova, Italy. Currently he is the Research and Development Manager of the Power Network Demonstration Centre at the University of Strathclyde in Glasgow, Scotland. His research interests include power system protection, automation and control.

Campbell Booth received the B.Eng. (Hons) and $\mathrm{PhD}$. degrees from the University of Strathclyde, Glasgow, U.K. Currently, he is a Senior Lecturer within the Institute for Energy and Environment at the University of Strathclyde in Glasgow, Scotland. His research interests include power system protection and plant condition monitoring.

Adam Dyśko (M’06) received the M.Sc. degree from the Technical University of Łódź, Poland, and the Ph.D. degree from the University of Strathclyde, Glasgow, U.K., in 1998. Currently, he is a Lecturer in the Department of Electronic and Electrical Engineering. His main interest areas are power system modeling, protection, and power quality. 